\title{
Nuevos registros de Hippidion (Mammalia, Perissodactyla) en el Pleistoceno tardío de la Provincia de Buenos Aires, Argentina
}

\author{
New record of Hippidion (Mammalia Perissodactyla) from the late \\ Pleistocene of Buenos Aires province, Argentina
}

\author{
J.L. Prado' ${ }^{1}$, R. Bonini², M.T. Alberdi ${ }^{3}$ A. Scanferla ${ }^{4}$, L.H. Pomi², E. Fucks ${ }^{5}$
}

\section{RESUMEN}

En este estudio se describen dos nuevos cráneos referidos a Hippidion principale (Lund) y varios elementos del esqueleto apendicular referidos a $H$. devillei procedentes de dos localidades clásicas de la provincia de Buenos Aires, las márgenes de los ríos Salado y Quequén Grande. Para la identificación taxonómica de los restos apendiculares, se realizó un análisis multivariante en el que se incluyeron los metacarpianos y falanges descritos y los registrados en varias localidades de Argentina y Brasil. Para la determinación de los cráneos se realizó un estudio comparativo con otros cráneos de diversas localidades. Estos hallazgos incrementan el registro de Hippidion en América del Sur y aportan nuevas evidencias sobre la cronología de sus especies en Argentina. Se realizó una datación por AMS para el ejemplar de Río Salado (MHM-P54) que ha suministrado una edad ${ }^{14} \mathrm{C} 14120 \pm 50 \mathrm{BP}$. En general, los sedimentos portadores en ambas localidades son referibles a la Edad Lujanense, Biozona de Equus (Amerhippus) neogeus.

Palabras clave: Extinción, Mammals, Equidae, Hippidion, Pleistoceno tardío, Argentina.

\section{ABSTRACT}

This study describes two new skulls referred to Hippidion(Lund) and various elements of the appendicular skeleton referred to $H$. devillei from two classic localities from Buenos Aires province: the Salado and Quequén Grande Rivers. For taxonomic identification of the appendicular remains was performed a multivariate analysis that included the metacarpals and phalanges described and several remains recorded in various locations in Argentina and Brazil. For the determination of the skulls was made a comparative study with other skulls from different localities. These data increasing the record of Hippidion in South America and provide new evidence about the chronological distribution of two species of Hippidion. One radiometric data was obtained by the method of AMS dating to the skull from Salado River (MHM-P54) about a ${ }^{14} \mathrm{C}$ age $14120 \pm 50 \mathrm{BP}$. In general, bearing sediments at both localities are referable to the Lujanian Age and the Biozone of Equus (Amerhippus) neogeus.

Keywords: Extinction, Mammals, Equidae, Hippidion, Latest Pleistocene, Argentina

\footnotetext{
${ }^{1}$ INCUAPA, UE CONICET. Universidad Nacional del Centro. Del Valle 5737. B7400JWI Olavarría (Argentina). Email: jprado@soc.unicen.edu.ar

2 División Paleontología de Vertebrados, Facultad de Ciencias Naturales y Museo, Paseo del Bosque s/n, B1900FWA. La Plata, Argentina. Email: rbonin@fcnym.unlp.edu.ar

${ }^{3}$ Museo Nacional de Ciencias Naturales, CSIC. José Gutiérrez Abascal, 2. 28006-Madrid (Spain). Email: malberdi@mncn.csic.es

${ }^{4}$ CONICET, Museo Argentino de Ciencias Naturales "Bernardino Rivadavia". Av. Ángel Gallardo, 470. Buenos Aires, Argentina.

${ }^{5}$ Facultad de Ciencias Naturales y Museo y Ciencias Agrarias y Forestales, UNLP; Calle 64 NN$^{\circ}$ 3, (1900) La Plata; Argentina Email: efucks@fcnym.unlp.edu.ar
} 

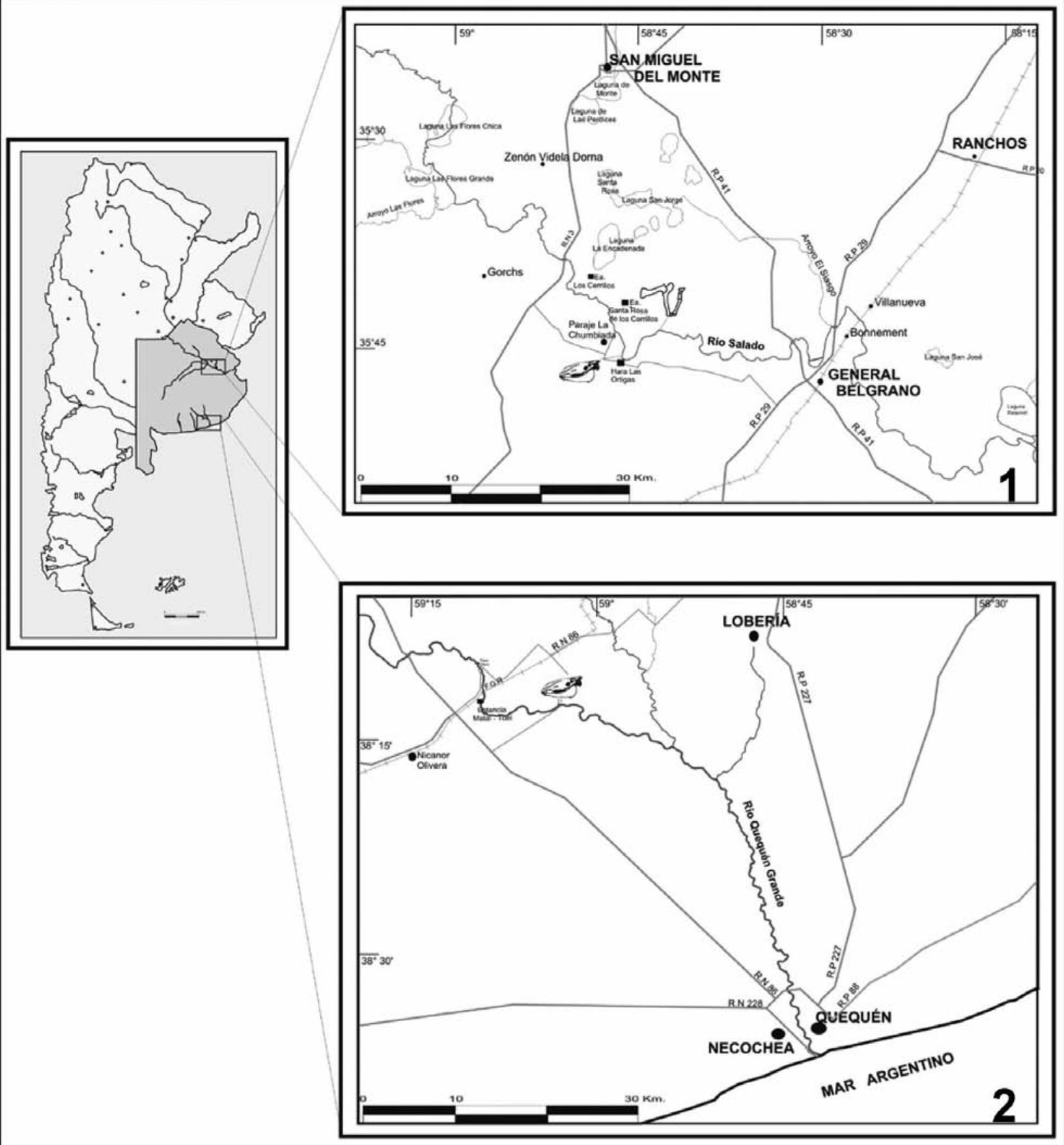

Fig. 1.-Ubicación geográfica de los sitios de hallazgo: 1, croquis curso inferior del río Salado. 2, croquis del curso inferior del río Quequén Grande. Los dibujos de cráneo y pata articulada representan los puntos del hallazgo de los restos.

\section{Introducción}

Los équidos fósiles son un componente importante en las comunidades de grandes mamíferos que poblaron las extensas llanuras durante el Cuaterna- rio en América del Sur. Sin embargo, tanto los caballos, como la gran mayoría de las especies de mamíferos de talla grande desaparecieron hacia finales del Pleistoceno. En lo que respecta a la región pampeana, se conoce que la extinción fue 
particularmente severa (Prado et al., 2001; Alberdi \& Prado, 2004; Barnosky \& Lindsay, 2010) y la mayor parte de las extinciones parecen concentrarse hacia fines del Pleistoceno. Sin embargo, la carencia de una cronología detallada no permite corroborar si la extinción fue tan rápida como en algunas regiones de América del Norte y si existe una sinergia con el arribo de las poblaciones humanas y/o el cambio climático. Este tipo de pregunta es crítica para poder entender como la sinergia de presiones independientes sobre la fauna puede o no puede multiplicar la intensidad de extinción.

En este sentido, el registro de caballos fósiles de la provincia de Buenos Aires ofrece la oportunidad de contrastar, con respecto a otras áreas, si las expectativas de la hipótesis de sinergia se sustentan. El registro de caballos fósiles de la provincia de Buenos Aires es significativo y proviene tanto de sitios paleontológicos como arqueológicos pero la mayoría de ellos son datos antiguos con poco control estratigráfico y cronológico (Prado \& Alberdi, 1999, 2010).

En este estudio se dan a conocer los restos de caballos fósiles procedentes de dos localidades clásicas de la provincia de Buenos Aires, las márgenes de los ríos Salado y Quequén Grande (Figura 1). Estos hallazgos tienen la importancia de aumentar el número de localidades con restos de Hippidion en esta provincia, así como establecer una cronología precisa que contribuye a entender las posibles causas que llevaron a la extinción. También, mediante el análisis morfológico y biométrico de los restos descriptos se analizan las adaptaciones paleoambientales de estos hallazgos.

\section{Contexto geológico y cronológico}

\section{Geología regional}

Fidalgo et al. $(1973,1975,1991)$ revisaron la nomenclatura estratigráfica del Cuaternario final de la Región Pampeana. Basado en la evidencia geomorfológica, así como el análisis de textura y composición de los depósitos del Pleistoceno y el Holoceno, propusieron dos unidades estratigráficas: la Formación Luján con dos miembros (Guerrero y Río Salado) y la Formación La Postrera. La primera es de origen fluvio-lacustre, y el segundo es de origen eólico. Los depósitos eólicos que cubren todo la Región Pampeana comprenden loess, arenas muy finas estratificadas y campos de dunas (Zárate \&
Blasi, 1993). Los análisis sedimentológicos del material eólico (Bidart, 1988; Zárate \& Blasi, 1991) sugieren que el volcanismo en la región andina fue el proceso predominante de la formación de partículas, específicamente, como generador de depósitos piroclásticos que fueron reelaborados por procesos erosivos fluviales, eólicos y glaciares. Gardenal (1986) describe cuatro grupos principales de geoformas eólicas en la parte occidental de la provincia de Buenos Aires. La calibración de estos episodios es difícil. De acuerdo con Zárate \& Blasi (1991), los dos primeros episodios corresponden al final del Pleistoceno, mientras que el tercero se produjo en el Holoceno medio y el último en tiempos históricos. Por el contrario, la Formación Luján está representada por los depósitos de llanura de inundación que se encuentran ampliamente desarrollados a lo largo de los principales sistemas fluviales en la provincia de Buenos Aires (Fidalgo et al., 1973). La capa inferior de esta Formación corresponde en gran parte a los depósitos fluviales (los miembros La Chumbiada y Guerrero), mientras que las capas superiores son esencialmente lacustres (miembro Río Salado).

Dos unidades edafoestratigráficas fueron reconocidas en la cuenca baja del río Salado. El primero, llamado suelo Puesto Callejón Viejo (fechado por ${ }^{14} \mathrm{C}$ en $10,070 \pm 140 \mathrm{BP}$ en carbonato pedogenético; $9710 \pm 100 \mathrm{BP}$, en caracoles terrestres y $8940 \pm 130$ de BP en los componentes orgánicos del suelo) que se desarrolla en la parte superior del Miembro Guerrero, y el segundo, denominado paleosuelo Puesto Berrondo, desarrollado en la parte superior del Miembro Río Salado (Fidalgo et al., 1991; Bonadonna et al., 1995). El último paleosuelo indica un régimen mayor de humedad que podría coincidir con un momento más alto del nivel del mar, manifestado por la ingresión marina registrada en torno a 5000-6000 BP (Schnack, 1987; Zárate \& Flegenheimer, 1991). Anteriormente a ésta, otra ingresión marina asociada al MIS 5e, denominada Formación Pascua, ha sido descripta para la zona costera (Fidalgo et al., 1973; Isla et al., 2000; Schnack et al., 2005).

\section{Geología local}

\section{Perfil 1 Río Salado}

El perfil presentado en la Figura 2A ilustra la sucesión sedimentaria expuesta en la margen derecha del río Salado en las proximidades del paraje 


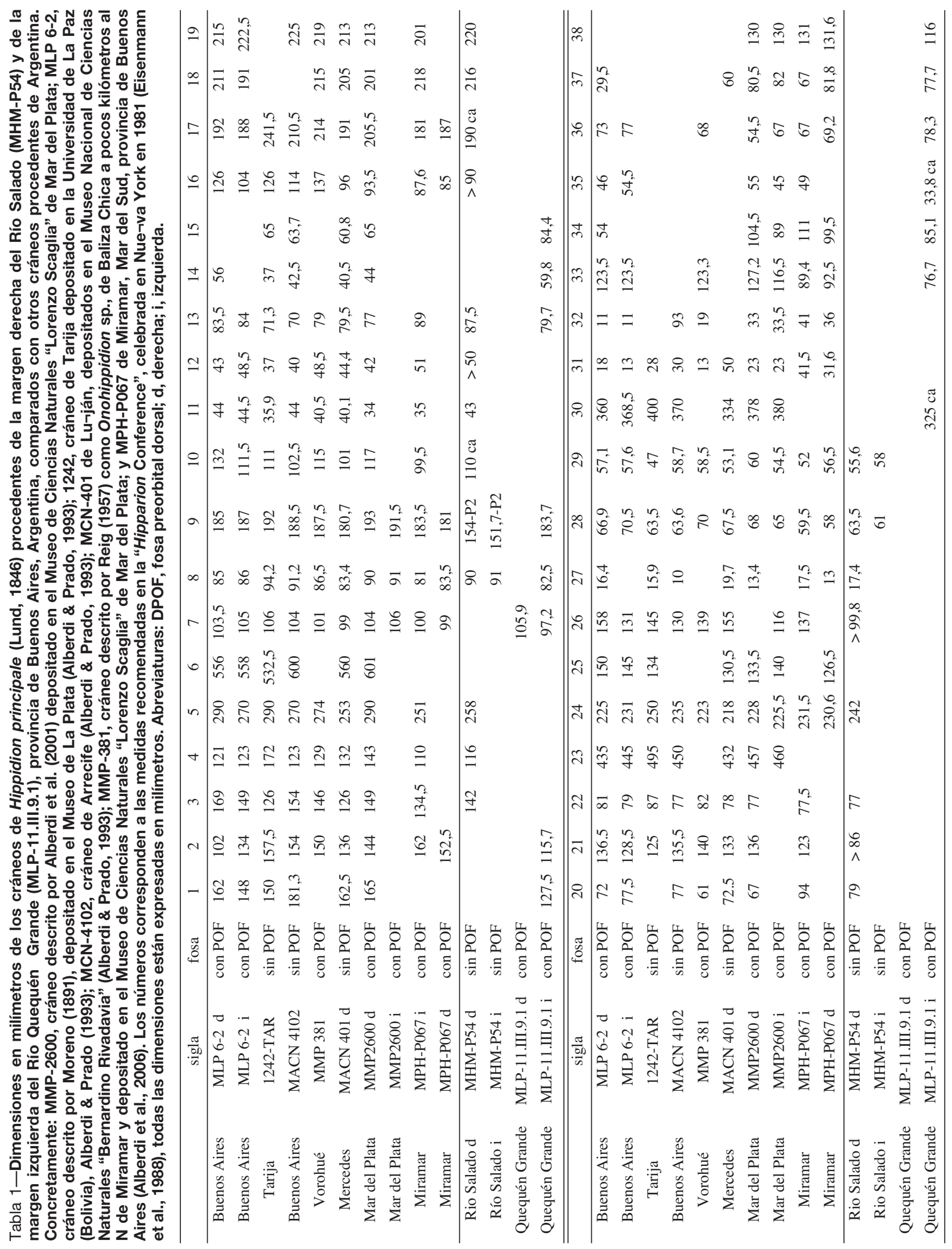



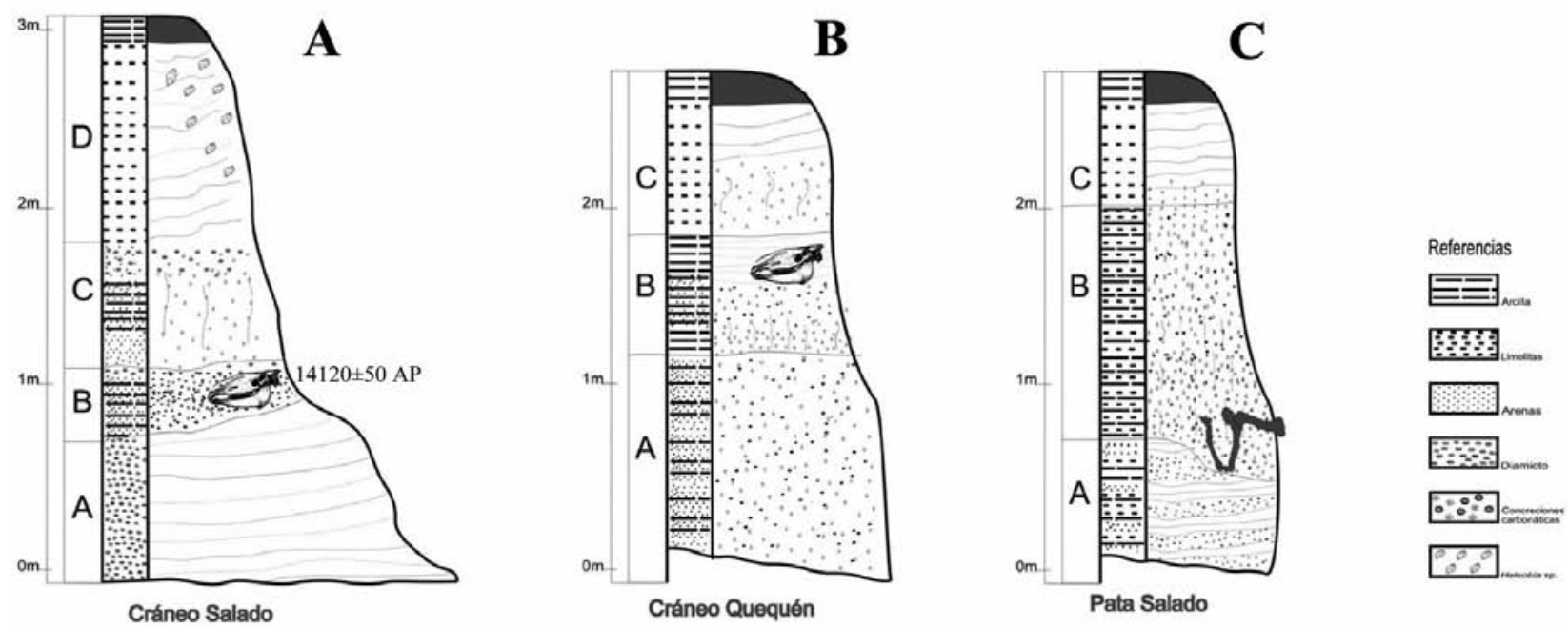

Fig. 2.-(A) Perfil esquemático de la margen derecha del Río Salado, a los 3544' 54"' S / 58 45' 53'” O, partido de General Belgrano, provincia de Buenos Aires, Argentina sitio del hallazgo del ejemplar MHM-P 54. (B) Perfil esquemático de la margen izquierda

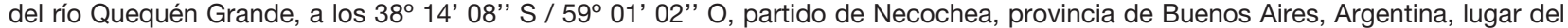

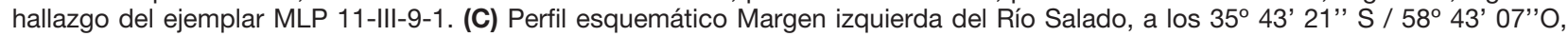
partido de San Miguel del Monte, provincia de Buenos Aires, Argentina, lugar del hallazgo del ejemplar MLP 01-V-1-66.

“La Chumbiada" (354 44' 54" S / 58 45'53" O). El mismo está representado por una sucesión de aproximadamente tres metros de potencia, de sedimentos de origen principalmente fluvial-lacustre.

NIVEL A (Base) - Está representado por los sedimentos que conforman el piso del canal, presenta una potencia de aproximadamente $1 \mathrm{~m}$ de altura, compuesto por un sedimento diamíctico de color castaño, resistente pero friable en el que se observa una tenue estratificación, asignable al Miembro La Chumbiada de la Formación Luján. Los clastos irregulares están compuestos por sedimentos de la Fm Pampeano y escasos de CO3Ca. El poco grado de redondeamiento y resistencia, y la similitud con los sedimentos de la Fm Pampeano, permitirían inferir un escaso transporte.

NIVEL B - representado por facies areno arcillosas, de color gris oscura a negra de unos 50 $\mathrm{cm}$ de espesor, que afloran aproximadamente $50 \mathrm{~cm}$ más por debajo de la superficie. Presenta concentraciones de concreciones de tosca localizados en niveles más pequeños. Este nivel es portador del cráneo de Hippidion principale que se describe en este articulo (ejemplar MHM-P54). De este nivel, también se recuperaron otros restos que se asignan tentativamente a Smilodon, Doedicurus y Megatherium.

NIVEL C - está compuesto por arena castaño verdosa con lentes de la facies inferior, presentán- dose la parte superior muy mezclada. Por encima se observan unos $50 \mathrm{~cm}$ de arena limosa castaña y grisácea con concreciones de carbonato de calcio aunque no muy resistentes

NIVEL D - está representado por aproximadamente $1,5 \mathrm{~m}$ de sedimentos limosos, resistentes, laminados, con restos antrópicos de génesis fluvial (Aluvio). Por encima de este depósito se desarrolla el suelo actual.

A aproximadamente $500 \mathrm{~m}$ aguas arriba del perfil esquematizado y suprayaciendo al nivel $\mathrm{C}$, se observan $1,5 \mathrm{~m}$ de sedimentos de color gris amarillento y granulometría limo-arcilloso, con gran cantidad de caracoles dulceacuícolas asignados al género Heleobia.

\section{Perfil Río Quequén Grande}

Este perfil está ubicado en las coordenadas $38^{\circ} 14^{\prime}$ $08^{\prime \prime} \mathrm{S}$ y $59^{\circ} 01^{\prime} 02^{\prime \prime} \mathrm{O}$ y está compuesto por una sucesión sedimentaria de aproximadamente $2,50 \mathrm{~m}$ de potencia, en la cual pueden observarse al menos 3 niveles diferenciados por litología y color, los que pueden ser atribuidos a un origen fluvial (Figura 2B).

Nivel A - La base de la sucesión no está expuesta, por encima del nivel de agua se observa un primer nivel de aproximadamente 1,20 m, compuesto por sedimentos limosos a arenosos de coloración verdosa. 
Nivel B - nivel portador compuesto por sedimentos arcillosos en la base y limosos a arcillosos hacia la parte media culminando con sedimentos finos laminados de color verde hacia el techo. En los primeros centímetros del nivel, por debajo del cráneo de Hippidion, se observan laminaciones de arcillas blanquecinas. Por encima del anterior y constituyendo el sedimento que cubría al ejemplar MLP 11-III-9-1, se observan sedimentos de granulometría areno-arcilloso de colores verdosos a grisáceos, rematando en laminados verdosos muy consolidados.

Nivel C - hacia el techo de la columna se observa paquete sedimentario de aproximadamente 50 $\mathrm{cm}$ de potencia, compuesto por sedimentos limosos de color verdoso sin una estratificación definida. Por encima de este nivel se desarrollan aproximadamente $1 \mathrm{~m}$ de sedimentos limosos de color gris amarillento de origen fluvial (Aluvio) culminando la columna con el desarrollo del suelo actual.

\section{Perfil 2 Río Salado}

Este perfil se realizó en la margen izquierda del Río Salado (Figura 2C), aguas abajo del anterior ( $\left.35^{\circ} 43^{\prime} 21^{\prime \prime} \mathrm{S} / 58^{\circ} 43^{\prime} 07^{\prime \prime} \mathrm{O}\right)$, ya en el partido de San Miguel del Monte. En este perfil se reconocen tres unidades. El paquete sedimentario posee una potencia de unos $2,5 \mathrm{~m}$.

NIVEL A - La base de la secuencia está compuesta por sedimentos limo-arcillosos pardos rojizos, con algunas intercalaciones arenosas finas, que podrían ser asignadas al Miembro La Chumbiada. Su límite inferior no pudo ser observado por estar cubierta por las aguas del río.

NIVEL B - posee una potencia de aproximadamente 1,15 metros compuesto por sedimentos limo - arcillosos de coloración gris verdosa que probablemente correspondan al Miembro Guerrero en el sentido de Fidalgo et al. (1973). En este nivel aparece una extremidad anterior derecha completa (ejemplar MLP-01.V.1.66) en conexión anatómica.

NIVEL C - el límite inferior de este nivel no es neto, sino que pasa de manera transicional a un sedimento limo - arenosos a arenas finas de color grisáceo.

\section{Situación Bioestratigráfica}

Florentino Ameghino, estableció el estándar de referencia para el Cenozoico continental de Améri- ca del Sur y propuso una secuencia de edades agrupadas en unidades de orden superior (Ameghino, 1889, 1909).esquema de Ameghino, con sus subsecuentes modificaciones, sigue siendo hoy la base válida para establecer una cronología de alcance regional (Cione et al., 2007). Hasta la década de 1940 hubo una gran confusión debido a que los mismos nombres utilizaban para distinguir las unidades de roca, el tiempo y las unidades de faunas. Los trabajos de Simpson (1940) y Pascual et al. $(1965,1966)$ establecieron cierto orden al proponer un esquema de edades basadas en las secuencias continentales de mamíferos. Estas edades se establecieron mediante el conocimiento del grado evolutivo de las faunas, en la misma forma que lo hizo Ameghino. Si bien estas unidades no han sido reconocidas por el código estratigráfico han demostrado ser muy útiles como método de organización en el desarrollo de conceptos acerca de la estratigrafía y evolución de los mamíferos (Simpson, 1971; Pascual \& Ortiz Jaureguizar, 1990). El actual esquema bioestratigráfica reconoce las siguientes unidades bioestratigráficas para el Pleistoceno medio y superior: las Zonas de Mesotherium cristatus (Piso Ensenadense), la Zona de Megatherium americanum (que representaría el comienzo de la Edad Lujanense de Pascual et al., 1965), la Zona de Equus (Amerhippus) neogeus (finales de la Edad Lujanense de Pascual et al., 1965), y por último la Zona de Lagostomus maximus (Edad Platense de Tonni \& Cione, 1994; Cione \& Tonni, 1995).

Los sedimentos atribuidos al Piso/Edad Lujanense (120-8,5 ka AP) que son biostratigráficamente correlacionados con la Biozona de Equus (Amerhippus) neogeus (Cione \& Tonni, 2005), estarían representados, en la región pampeana, por la parte inferior de la Formación Luján la cual se halla en gran parte de los afloramientos de los valles fluviales de la provincia de Buenos Aires. La Formación Luján ha sido descripta como constituida por tres Miembros, desde el más antiguo: La Chumbiada (Dillon \& Rabassa, 1985), Guerrero y Río Salado (Fidalgo et al., 1973). La Formación Luján se encuentra representada tanto en la denominada pampa deprimida como en el área interserrana (Fidalgo et al., 1991). Los depósitos fluviales de ambas regiones muestran ciertas similitudes desde el punto de vista geológico, aunque parecen diferir en los modos tafonómicos involucrados (Pomi \& Scanferla, 2008). En este sentido, las asociaciones de la pampa deprimida (e.g. Luján, Río Salado) se caracterizan y difieren de lo observado en el área 
interserrana (e.g. Paso Otero, Quequén GrandeIndio Rico), por la presencia de esqueletos de meso y megamamíferos relativamente completos y articulados (Pomi, 2009).

\section{Geocronología}

La cuenca del Río Salado posee una gran riqueza de mamíferos fósiles pero su cronología radiométrica está poco estudiada. En comparación con otras cuencas se tiene una baja resolución cronológica. A partir de algunos restos óseos, gasterópodos y materia orgánica, Scanferla et al. (2011) han obtenido una serie de dataciones radiocarbónicas que abarcan el lapso entre aproximadamente los 9500 a 13.000 años AP. Los sedimentos portadores son atribuidos a la Formación Luján, pero estos autores no especifican de qué unidad litológica o miembro provienen las muestras. Los fechados reportados por Scanferla et al. (2011) son: $12.100 \pm 100$ (LP-2184, materia orgánica); $12.860 \pm 120$ (LP-2259, vértebra cervical de Hippidion principale); $13.400 \pm 200$ (LP2184, fémur de Smilodon populator); y $12.380 \pm 190$ (LP2568, pelvis de Doedicurus sp.).

En este artículo estamos reportando un nuevo fechado para el Miembro Guerrero de la Formación Luján, realizado sobre la raíz del P3 izquierdo del cráneo de Hippidion (MHM-P54), que ha suministrado una edad AMS de 14C 14.120 \pm 50 AP (Beta - 311032). Fucks et al. (2007, 2009) obtiene la edad de $8640 \pm 110$ AP para el techo del Miembro Guerrero en la misma cuenca (en la localidad de General Belgrano), a partir de restos de conchas de moluscos. Esta última edad permitiría prolongar el lapso de sedimentación del Miembro Guerrero (Fucks et al., 2007). Por su parte, los fechados radiocarbónicos disponibles en la cuenca del Río Quequén Grande permiten extender el rango temporal para el Miembro Guerrero de la Formación Luján entre los 21000 y 8500 AP (Tonni et al., 2003). En esta cuenca, los miembros La Chumbiada y Guerrero fueron referidos como "sector pardo" y "sector verde", respectivamente, por Prado et al. (1987). Para la parte superior de este sector pardo se han citado dos fechas, realizadas en carbonato y conchas de moluscos que dan edades de $26.930 \pm 860$ y 28.900 \pm 800 ños AP (Carbonari et al., 1992; Figiniet al., 1995), en tanto que para el miembro Río Saladohan obtenido diversos fechados sobre conchas de moluscos que arrojan una edad entre $10.840 \pm 380 \mathrm{y}$ $3395 \pm 307$ años AP (Bonadonna et al., 1995).
Por otra parte, en la cuenca del río Quequén Salado se han referido varias dataciones radiocarbónicas (Bonadonna et al., 1995), que acotan la edad del Miembro Guerrero entre $15.610 \pm 740$ y $15.370+780-710$ años AP y recientemente, Prado et al. (2012) dan a conocer una nueva datación que arrojó una edad de AMS ${ }^{14} \mathrm{C} 14.020 \pm 60$ años AP (GrA nr-47265) y se obtuvo del esmalte dentario de un M1 de Equus (Amerhippus) neogeus (MMH-98.09.97) proveniente del Miembro Guerrero en la localidad Cascada Paleolama. Recientemente, nuevos fechados dados a conocer por Toledo (2005), sobre conchas de moluscos provenientes del río Luján, extienden el rango temporal para el Miembro Guerrero hasta los 32.000 años AP. Este período envuelve dos tercios del Estadio Isotópico 2 y el comienzo del Estadio Isotópico 1 (Clapperton, 1993).

\section{Material y Método}

El material en estudio consiste en un cráneo incompleto de Hippidion (MHM-P54) procedente de la margen derecha del Río Salado, depositado en el Museo Histórico Municipal "Alfredo E. Mulgura" de General Belgrano. Un cráneo, también incompleto, de Hippidion (MLP-11.III.9.1) procedente del margen izquierdo del Río Quequén Grande y que está depositado en el Museo de La Plata. Una extremidad anterior derecha completa (MLP-01.V.1.66), en conexión anatómica, que consta del húmero, radio, ulna con el olécranon incluido, escafoide, lunatum, piramidal o triquetum, pisiforme, trapezoide, mágnum, unciforme, los metacarpianos II, III y IV, las falanges primera, segunda y tercera (1FIII, 2FIII, 3FIII), el sesamoideo distal y los 2 sesamoideos proximales, procedentes del margen izquierda del Río Salado y depositados en Museo de La Plata. Además hay un ejemplar que parece corresponder a un metacarpiano $\mathrm{V}$ que hace buen contacto con el metacarpiano IV, también depositado en el Museo de La Plata.

Tanto los cráneos como los restos de la extremidad anterior en estudio se han comparado morfológica y biométricamente con otros ejemplares procedentes de varias localidades de América del Sur depositados en distintos Museos e Instituciones. Se ha combinado la información de la anatomía comparada de cráneos y dientes, y se han aplicado análisis multivariantes a aquellos huesos del esqueleto apendicular más significativos a nivel taxonómico como son MCIII, 1FIII y 2 FIII. El análisis discriminante se ha realizado utilizando todas las variables juntas y dejando una muestra fuera (la muestra en estudio) frente a las poblaciones ya estudiadas representadas en unas matrices de 41 MCIII, 74 1FIII y 69 2FIII cuyos componentes ya están identificados. Para su descripción y análisis morfométrico, hemos seguido la nomenclatura y recomendaciones de la "Hipparion Conference" (Eisenmann et al., 1988). Para los análisis multivariantes se utilizó el Programa SPSS 15.0. Todas las dimensiones están expresadas en milímetros. 
Tabla 2.-Dimensiones en milímetros de los dientes superiores de los cráneos de Hippidion principale (Lund, 1846) procedentes de la margen derecha del Río Salado (MHM-P54) y de la margen izquierda del Río Quequén Grande (MLP-11.III.9.1), provincia de Buenos Aires, Argentina; Ls, longitud mesio-distal en superficie oclusal; As, anchura linguo-bucal en superficie ocusal; $\mathrm{H}$, altura del diente; LPr, Longitud mesio-distal del proatocono en superficie oclusal; FPr, forma del protocono; P, premolares superiores; $\mathrm{M}$, molares superiores.

$\begin{array}{cccccc}\text { Río Salado } & & & & & \\ \text { MHM-P54 } & \text { L } & \text { A } & \text { altura } & \text { LPr } & \text { FPr } \\ \text { P3 d } & 33 & 36 & 53 & 11,2 & \text { redondo } \\ \text { P4 d } & 31,1 & 33,1 & 52 & 8,3 & \text { redondo } \\ \text { M1 d } & 29 & 37,3 & 49 & 10,6 & \text { algo oval } \\ \text { M2 d } & 29 & 33 & 48,5 & 10 & \text { algo oval } \\ \text { M3 d } & 31 & 26,8 & 31,5 & 9,7 & \text { algo oval } \\ \text { P3 i } & 33 & 37 & 48 & 11 & \text { redondo } \\ \text { P4 i } & 30,8 & 35 & & 10,4 & \text { redondo } \\ \text { M1 i } & 31,7 & 36,5 & & 10 & \text { algo oval } \\ \text { M2 i } & 29,2 & 33,6 & & 9 & \text { algo oval } \\ \text { M3 i } & 30 & 27,7 & & 10 & \text { algo oval } \\ \text { Quequn Grande } & & & & & \\ \text { MLP-11.III.9.1 } & & & & & \text { m2 d } \\ \text { P3 } & 37,4 & 31 & & 11,25 & \text { oval abriendose } \\ \text { P3 d } & 31,6 & 34,8 & & 12,92 & \text { oval abriendose } \\ \text { P4 d } & 30,6 & 34,3 & & 12,92 & \text { más largado } \\ \text { P2 i } & 40,9 & 29,3 & & 13,04 & \text { oval abriendose } \\ \text { P3 i } & 30,2 & 32,1 & & 15,46 & \text { oval abriendose } \\ \text { P4 i } & 30,1 & 32,3 & & 14,29 & \text { más alargado } \\ \text { M1 i } & 25,9 & 29,8 & & 11,41 & \text { más alargado } \\ \text { M2 i } & 25,9 & 29,3 & & 10,93 & \text { más alargado } \\ \text { M3 i } & 32,2 & 27,7 & & 11,36 & \text { más alargado }\end{array}$

\section{Paleontología Sistemática}

Orden PERISSODACTYLA Owen, 1848

Familia EQUIDAE Gray, 1821

Subfamilia EQUINAE Gray, 1821

Tribu EQUINI Gray, 1821

Subtribu PLIOHIPPINA Prado \& Alberdi, 1996

Género Hippidion Owen, 1869

Hippidion principale (Lund, 1846)

Material: Cráneo (MHM-P54) y cráneo (MLP-11.III.9.1) (Figura 3).
Descripción: El cráneo (MHM-P54) de talla más bien grande, carece de fosa preorbital dorsal (DPOF) y a las series dentarias, P3-M3 derecha e izquierda, les faltan los P2 y toda la sínfisis. Le falta también el hueso nasal y la retracción de la hendidura nasal alcanza el nivel del mesostilo de M3, el saliente facial en el lado derecho está roto y en el izquierdo se inicia a nivel del parastilo de M1. El vómer en su parte más mesial está a nivel del protocono de M1. El orificio occipital tiene una anchura transversal de aproximadamente $33 \mathrm{~mm}$ y los cóndilos occipitales en sus bordes más externos se distancian $84,4 \mathrm{~mm}$. Parece corresponder a un animal adulto bastante maduro. Los premolares muy cuadrangulares y con el protocono redondo, en los molares algo más pequeño y el protocono algo más oval (dimensiones en las Tablas 1 y 2). De las series dentarias sólo hemos podido tomar la longitud de la serie molar M1-M3 derecha = 90 y M1-M3 izquierda $=91$, al faltar los $\mathrm{P} 2$ carecemos de las longitudes de las series premolares (P2-P4) y de las series totales (P2-M3) de los dientes yugales (dimensiones en Tabla 1).

El cráneo (MLP-11.III.9.1) de talla más bien grande con fosa preorbital dorsal (DPOF) y con la serie dentaria izquierda entera LP2-M3 = 183,70, LP2-P4 = 97,24 y LM1-M3 = 82,45, mientras que de la derecha sólo conserva la serie premolar LP2 P4 = 105,95. Este cráneo conserva la sínfisis con I2 e I3 derechos e izquierdos y los caninos muy robustos (carece de ambos I1); el diastema P2-C = 43,2 en el lado derecho y 41,37 en el izquierdo, y el diastema C-I3 es de 28,84 en el derecho y 29,4 en el izquierdo. A este cráneo le falta toda la parte distal y la parte lateral derecha, no quedan restos de las órbitas y la zona de máxima hendidura nasal también está rota (Figura 3). No disponemos de los huesos nasales y la retracción nasal parece alcanzar el nivel del parastilo de M2 (este punto está algo roto). La cresta facial en el lado izquierdo parece estar a nivel del parastilo del M1. Del vómer sólo conserva el borde izquierdo. Las dimensiones que se han podido tomar del cráneo están en la Tabla 1 junto a las dimensiones de los cráneos utilizados como comparación. El desgaste de los molares, sobre todos los premolares con los protoconos oval perdiéndose, indica que estamos ante un animal bastante viejo, más que el anterior (las dimensiones dentarias en la Tabla 2).

Comentarios: Las características morfológicas del cráneo del Río Salado y sobre todo su tamaño, muy grande y con dientes también grandes, corresponden a Hippidion principale. Las características morfológicas del cráneo del Río Quequén Grande y su tamaño podrían corresponde a un Hippidion de tamaño grande si bien las dimensiones de los dientes, muy grandes, son algo más pequeñas que las del primero.

Género Hippidion Owen, 1869

Hippidion devillei (Gervais, 1855)

Material: Una extremidad anterior derecha completa (MLP01.V.1.66), en conexión anatómica, que consta de húmero, radio, ulna con el olécranon incluido, escafoide, lunatum, piramidal o triquetum, pisiforme, trapezoide, mágnum, unciforme, los MCII, MCIII y MCIV, las falanges 1FIII, 2FIII y 3FIII, el sesamoideo distal y los 2 sesamoideos proximales

Descripción: En los restos de esta extremidad (MLP01.V.1.66) se observa que las dimensiones del húmero, radio y ulna (Tablas 3 y 4) se sitúan entre los rangos obtenidos para $H$. principale e $H$. devillei. Para la discriminación taxonómica del resto de la extremidad, concretamente (MCIII, 1FIII y 2 FIII), los hemos comparado, mediante un análisis discriminante, con los restos ya conocidos de otras localidades de América del Sur. 


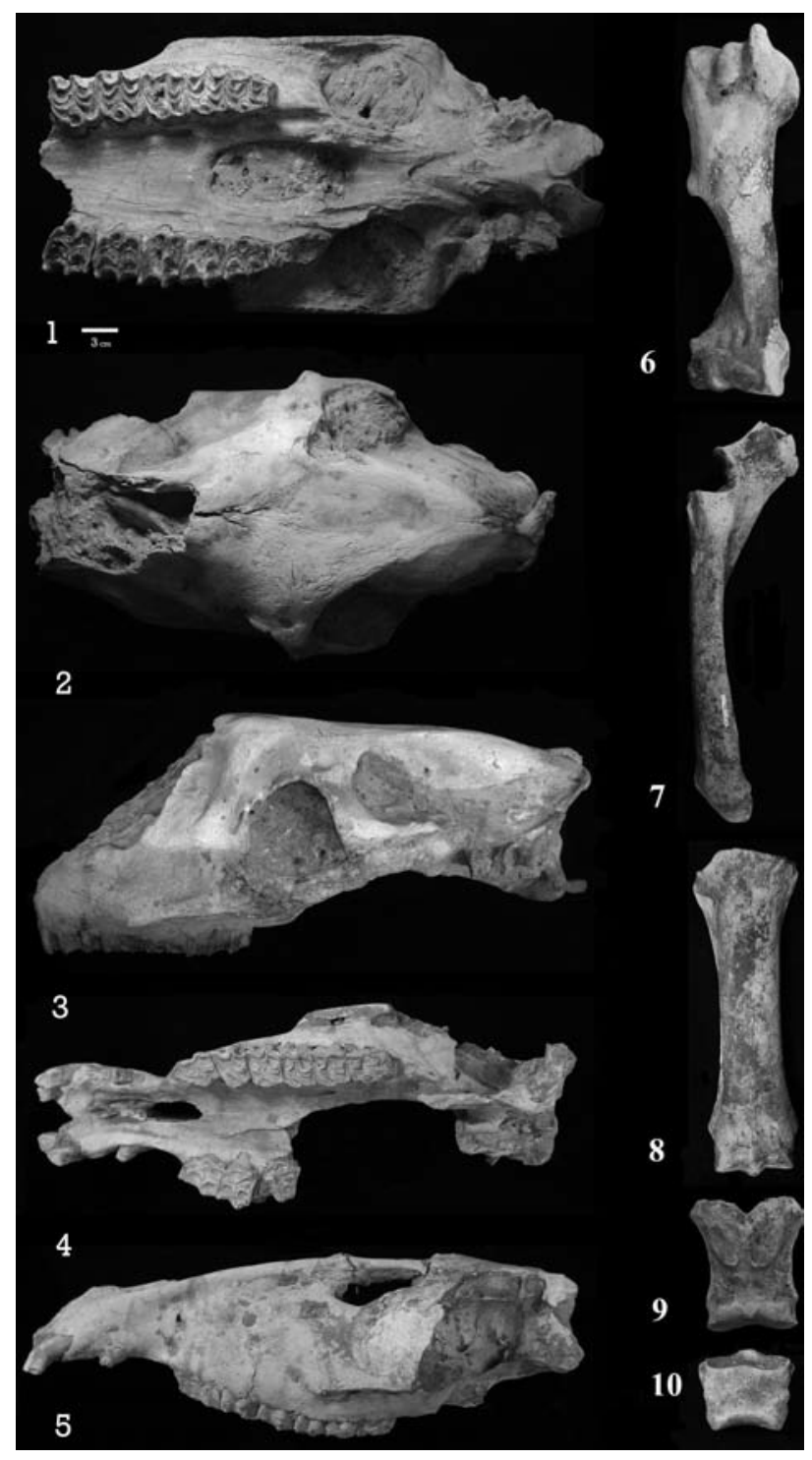

Fig. 3.-1, cráneo de Hippidion principale (MHM-P54) en vista ventral, procedente de la margen derecha del Río Salado, partido de General Belgrano, provincia de Buenos Aires, Argentina. Depositado en el Museo Histórico Municipal "Alfredo E. Mulgura" de General Belgrano; 2, el mismo en vista dorsal; 3, el mismo en vista lateral; 4, Cráneo de Hippidion principale (MLP-11.III.9.1) en vista ventral, procedente del margen izquierdo del Río Quequén Grande, partido de Necochea, provincia de Buenos Aires, Argentina. Depositado en el Museo de La Plata; 5, el mismo en vista lateral; 6 , húmero derecho en vista anterior, procedente de la margen izquierda del Río Salado, partido de San Miguel del Monte, provincia de Buenos Aires, Argentina; 7, radio y ulna derecha en vista lateral, procedente de la margen izquierda del Río Salado, partido de San Miguel del Monte, provincia de Buenos Aires, Argentina; 8, MCIII derecha en vista anterior, procedente de la margen izquierda del Río Salado, partido de San Miguel del Monte, provincia de Buenos Aires, Argentina; 9, 1 FIII derecha en vista posterior, procedente de la margen izquierda del río Salado, partido de San Miguel del Monte, provincia de Buenos Aires, Argentina; 2FIII derecha en vista anterior, procedente de la margen izquierda del Río Salado, partido de SanMiguel del Monte, provincia de Buenos Aires, Argentina.

\section{Análisis discriminante}

Con los restos especificados más arriba hemos realizado un análisis discriminante dejando uno fuera, es decir comparando los restos de la localidad en estudio con los conocidos de otras localidades ya estudiadas. Así, en el caso del MCIII éste se incluye en la base de datos de restos ya clasificados. En el análisis discriminante el ejemplar del MCIII de Río Salado se asocia claramente con los restos de $H$. devillei (con una clasificación correcta del $100 \%$ y el $78,8 \%$ por validación cruzada incluyendo todas las variables junta, y del $88,6 \%$ y $85,7 \%$ respectivamente con el método de Mahalanobis); mientras que la 1 FIII quedan entre las de $H$. principale con una clasificación correcta del $83,6 \%$ y $78,1 \%$ incluyendo todas las variables juntas, y del $76,7 \%$ y $75,3 \%$ por el método de Mahalanobis; y en el caso de la 2FIII ésta queda agrupada con $H$. devillei con una clasificación correcta del $100 \%$ y $95,6 \%$ para todas las variables juntas y $98,5 \%$ y $94,1 \%$ para el método de Mahalanobis (Figura 4). Se debe tener en cuenta que entre las falanges en general, y en las primeras en particular, las diferencias que suele haber entre las falanges anteriores y las posteriores es más acusada entre las primeras falanges que entre las segundas, unido a las escasas diferencias que presentan las falanges entre las distintas especies de Hippidion. Todo ello, enmascara, en parte, las diferencias de las primeras falanges entre las distintas especies de este género. De hecho si se observa atentamente la figura 4 se puede observar un mayor solapamiento entre las primeras falanges de las tres especies de Hippidion, que están mucho mejor definidas en los diagramas del MCIII y la 2FIII. El húmero y el radio son más grandes que los de Calama, y parecen muy próximos a los datos que disponemos de $H$. principale, si bien el número de restos de de huesos largos completos de que disponemos son bastante escasos. En cuanto a los huesos del carpo estos no presentan grandes diferencias entre las tres especies.

\section{Discusión}

Los caracteres morfológicos dentales de ambos cráneos son claramente de Hippidion, uno de la margen derecha del río Salado y el otro de la margen izquierda del Río Quequén Grande. Las mayores diferencias entre las distintas especies de este género están precisamente en su tamaño, claramente diferentes, más acusado en la dentición, el cráneo y 
Tabla 3.-Dimensiones (en $\mathrm{mm}$ ) de los huesos largos de la extremidad anterior derecha de Hippidion devillei (Gervais, 1855) procedentes de la margen izquierda del Río Salado (MLP-01.V.1.66): húmero, radio, ulna y metacarpiano del tercer dedo (MCIII) y posible quinto (MCV). Los números indican las dimensiones recomendadas en la "Hipparion Conference" (Eisenmann et al., 1988). Todas las dimensiones están en milímetros.

\begin{tabular}{|c|c|c|c|c|c|c|c|c|c|c|c|c|c|c|c|c|c|c|}
\hline Hueso & localidad & sigla & med 1 & med 2 & med 3 & med 4 & 4 med 5 & med 6 & $\operatorname{med} 7$ & 7 med 8 & med 9 & 9 med 10 & 0 med 11 & $1 \mathrm{med} 12$ & med 13 & $13 \mathrm{med}$ & $14 \mathrm{me}$ & ed 16 \\
\hline humero $\mathrm{d}$ & Rio Salado & MLP-01.V.1.66 & 320 & 289,5 & 38,5 & 49 & 103,5 & 112 & 87 & 82,2 & 87 & 38,8 & 52 & & & & & \\
\hline radio $\mathrm{d}$ & Rio Salado & MLP-01.V.1.66 & 314 & 298 & 47 & 29,4 & 81,3 & 42 & 92 & 68,3 & 40,4 & 85,4 & 29,2 & 17,8 & & & & \\
\hline ulna d & Rio Salado & MLP-01.V.1.66 & 390 & 86 & & 63 & & & & & & & & & & & & \\
\hline McIII d & Rio Salado & MLP-01.V.1.66 & 190 & 185 & 36,1 & 28,7 & 52,8 & 33,6 & 42,7 & 15 & 5,1 & 55,7 & 49,5 & 37,6 & 30 & 32 & & 11 \\
\hline McII d & Rio Salado & MLP-01.V.1.66 & 136 & & 19 & 13 & & & & & & & & & & & & \\
\hline McIV d & Rio Salado & MLP-01.V.1.66 & 118 & & 20 & 12 & & & & & & & & & & & & \\
\hline $\mathrm{McV} d ?$ & Rio Salado & MLP-01.V.1.66 & 19 & & & & 12 & 11,1 & & & & & & & & & & \\
\hline
\end{tabular}

Tabla 4.-Dimensiones (en $\mathrm{mm}$ ) de los huesos cortos de Hippidion devillei (Gervais, 1855) procedentes de la margen izquierda del Río Salado (MLP-01.V.1.66). Los números de las medidas indican las dimensiones recomendadas en la "Hipparion Conference" (Eisenmann et al., 1988) para las falanges. En el caso de los huesos del carpo que tienen forma más o menos rectangular se toman las tres medidas máximas; mientras que en el resto se toma la altura externa y la longitud transversal y la profundidad de la articulación superior y de la inferior. Todas las dimensiones están en milímetros.

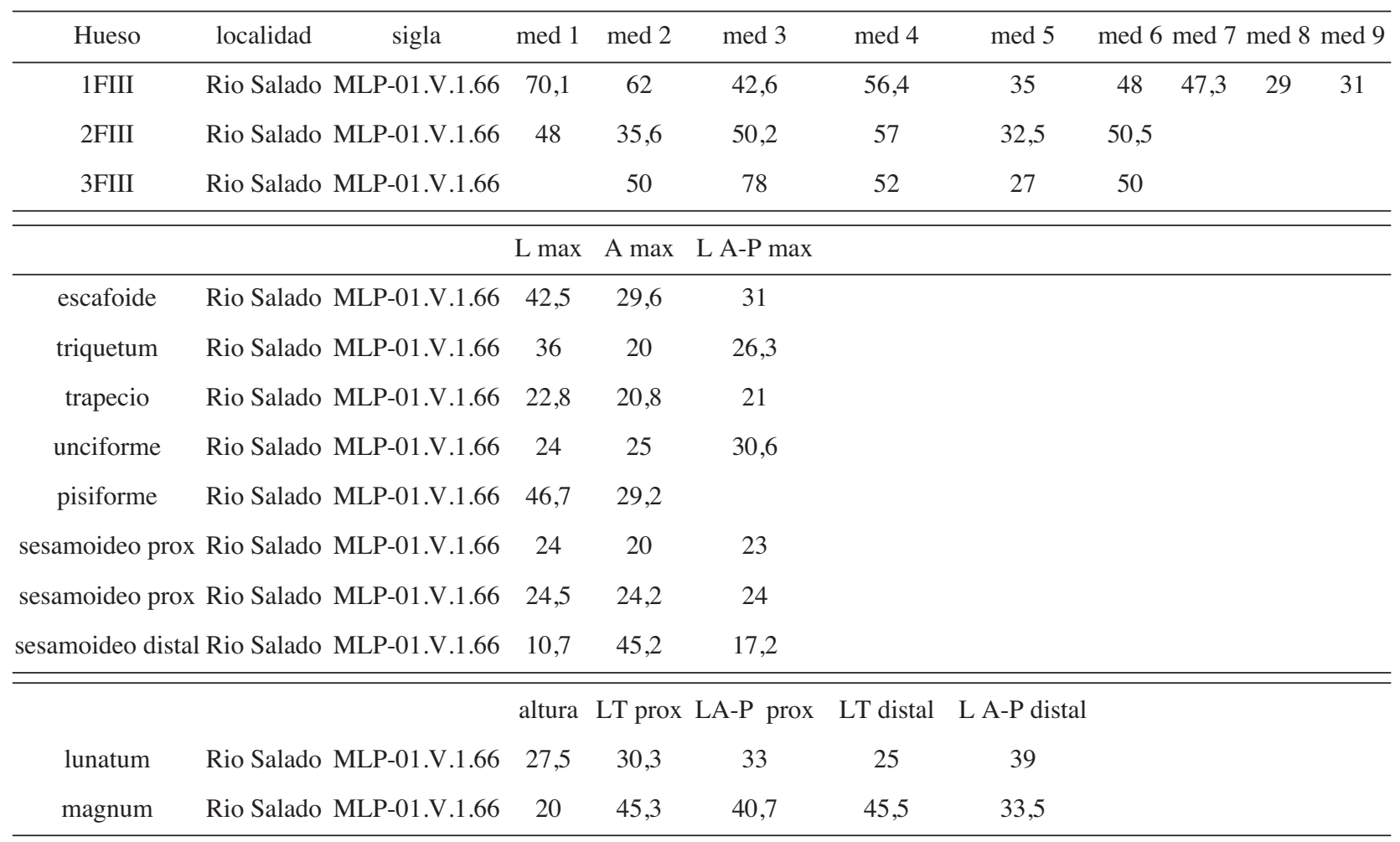

sobre todo en el acortamiento de los metápodos que en otros restos del esqueleto apendicular (Alberdi \& Prado, 1993). Los cráneos estudiados en este trabajo son claramente de gran tamaño y uno corresponde a un animal maduro mientras que el otro es bastante más viejo (ejemplar de Quequén Grande) donde el dibujo de la superficie oclusal de los dientes inicia su desaparición. El de río Salado carece de fosa preor- 
bital (DPOF) mientras que el de Quequén Grande la posee, una fosa no muy grande pero clara. El porqué de la presencia de esta fosa en unos cráneos si y en otros no, no está todavía clara y ha suscitado una fuerte controversia que repercute en la sistemática de los hippidiformes sudamericanos. En efecto, a lo largo de estos años ha persistido una diferencia de opinión entre dos de los autores (MTA y JLP) y Bruce MacFadden sobre la validez del género Onohippidium y la utilidad desde el punto de vista sistemático de la morfología de la DPOF (MacFadden, 1997, 1998; Alberdi \& Prado, 1998). Los trabajos de Alberdi (1987), Alberdi \& Prado $(1993,1998)$ y Prado \& Alberdi (1996) concluyen que Hippidion es el único género válido y endémico de América del Sur. MacFadden $(1997,1998)$, sin embargo, considera que deben ser válidas tanto Hippidion como Onohippidium. Este último género fue nominado por Moreno (1891) sobre la base de la presencia de DPOF. La respuesta de MacFadden (1998) a nuestra réplica sobre su reivindicación del género Onohippidium se redujo a decir que si no se acepta la utilidad de la forma y tamaño de la DPOF en la taxonomía de los hipidiformes, tienen razón Alberdi \& Pardo (1998) y entonces es cierto que solo hay un género: Hippidion, pero si se acepta la utilidad del carácter DPOF en los estudios morfológicos de los équidos, entonces tiene razón MacFadden (1998).

En lo que respecta a los ejemplares craneales analizados consideramos que, la morfología del cráneo, la dentición y el tamaño es el mismo y solo varía la zona de la base del nasal ligada precisamente a la presencia o no de la fosa. El hecho de que haya otro grupo de animales que presentan estructuras similares en los machos ligadas al dimorfismo sexual nos inclina a pensar en la posibilidad de que en este grupo se diera una situación equivalente, o que pudiera estar ligado a algún factor ambiental. Consideramos que las diferencias que hemos encontrado entre ambos tipos de cráneos corresponden más a la diferencia de edad de los distintos ejemplares que a la presencia o no de una DPOF. En referencia a los cráneos utilizados como elementos comparativos hay que considerar que el ejemplar de Mar del Sud es bastante viejo (Alberdi et al., 2006), mientras que el ejemplar de Mar del Plata era bastante joven (Alberdi et al., 2001), este ejemplar de Quequén Grande también es viejo y quizás incluso más que el de Mar de Sud; otros cráneos con los que hemos comparado el ejemplar de Río Salado corresponden a individuos adultos más o menos viejos; la edad queda claramente reflejada en la superficie oclusal
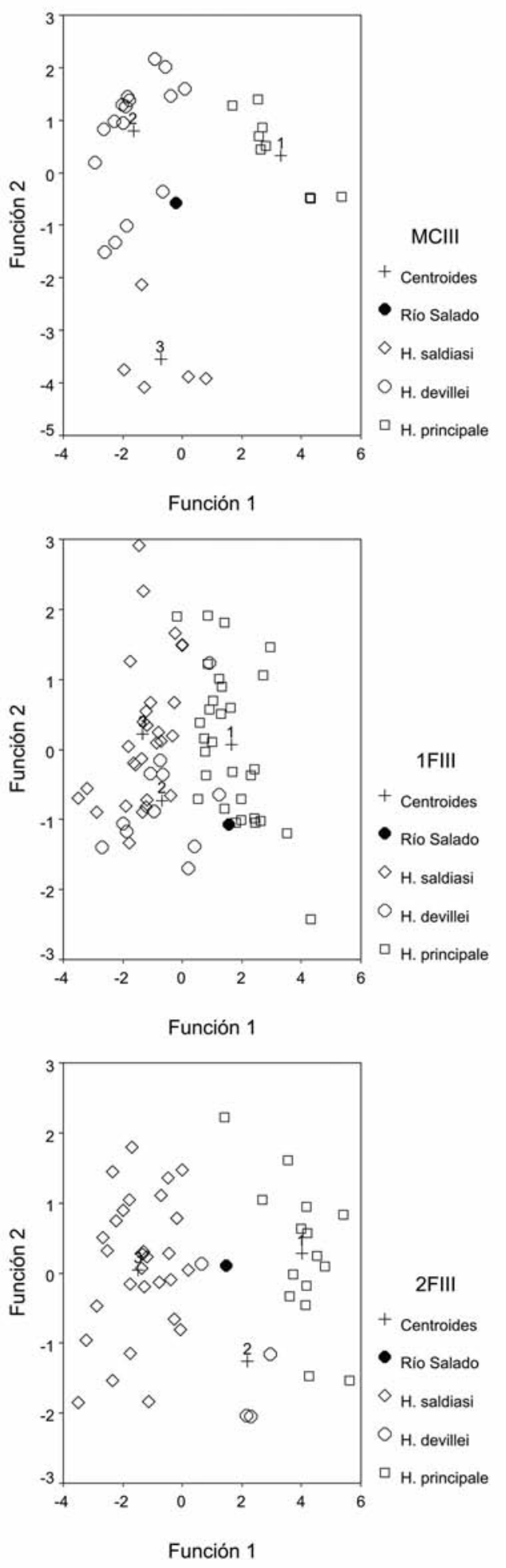

Fig. 4.-Diagramas de los análisis discriminantes de MCIII, 1FIII y 2FIII de Hippidion principale procedentes de la margen izquierda del Río Salado, partido de San Miguel del Monte (provincia de Buenos Aires, Argentina) comparados con los restos de las distintas especies de Hippidion de América del Sur. 1, 2 y 3 son los centroides correspondientes a las tres especies de Hippidion. 
de los dientes. El ejemplar de Quequén Grande sería de una edad aproximada al de Mar del Sud, aunque un poco más viejo, las longitudes de sus series dentarias son muy iguales (ver en Tabla 1 la longitud P2-M3 del cráneo de Miramar que es el ejemplar de Mar del Sud). Como vemos en la Tabla 1, la mayoría de las dimensiones son similares (las diferencias se pueden considerar debidas a la variabilidad individual) y las mayores diferencias corresponden a la zona de la DPOF de la cual carece el cráneo de río Salado pero está presente en el de Quequén Grande, si bien la zona está bastante rota. En suma, consideramos que ambos cráneos son claramente referibles a Hippidion principale.

En el caso de la extremidad anterior derecha que se encontró articulada y en conexión anatómica se observa, sobre todo húmero y radio, que son de tamaño claramente mayores que los de $H$. saldiasi de Calama, y el radio es menor que los de $H$. principale de Tarija y el húmero es similar al de $H$. principale de Tarija (tener en cuenta que los restos enteros y articulados son muy escasos). En el caso del MCIII, bastante acortado, el análisis discriminante lo agrupa con los restos de $H$. devillei conocidos (Figura 4), igualmente ocurre con la 2FIII, mientras que en el caso de la 1FIII el análisis discriminante lo agrupa con los restos de $H$. principale. Examinando en detalle las dimensiones del conjunto de las falanges observamos que el ejemplar de río Salado se sitúa en los límites del rango de $H$. devillei. Como ya nos pasó en el estudio de los restos de Toca dos Ossos (Brasil, Alberdi et al., 2003) la presencia de $H$. principale e $H$. devillei en un mismo yacimiento dificulta la identificación correcta de algunos restos del esqueleto apendicular (huesos largos, del carpo y tarso), e incluso las diferencia entre las falanges centrales son bastante reducidas, mientras que las diferencia con los de $H$. saldiasi son más claras. Sin embargo, los metápodos en general, tanto metacarpos como metatarsos, presentan diferencias claramente significativas. Así el análisis discriminante de este MCIII de río Salado, reducido en longitud, así como el análisis comparativo con otros metacarpianos de Hippidion de América del Sur permite su asignación clara a $H$. devillei.

\section{Consideraciones finales}

Los équidos son uno de los grupos más característicos del Pleistoceno sudamericano. El nombre de Hippidion, creado por Owen en 1869, junto con el de Equus, fue citado en numerosas localidades de Argentina y otros países de América del Sur. El primer representante de Hippidion fue descrito a partir de un único molar de una brecha antigua de la Cueva de Lagoa Santa (Brasil) y figurado por Lund en 1846. Alberdi \& Prado (1993) y Prado \& Alberdi (1996) consideran que este género es endémico en América del Sur donde se expande desde el Plioceno superior al Pleistoceno final (Alberdi \& Prado, 1993) y geográficamente se distribuye en gran parte de América del Sur, principalmente en Argentina, Bolivia, Brasil, Chile, Colombia, Perú y Uruguay.

A la vista de los datos existentes y las discusiones previas, podemos identificar los restos craneales procedentes de río Salado y el de Quequén Grande descritos aquí como Hippidion principale, mientras que la extremidad anterior derecha que provienen del otro afloramiento de río Salado correspondería a Hippidion devillei. De acuerdo a la edad obtenida, el ejemplar de río Salado (MHM-P54) arrojó una edad AMS de ${ }^{14} \mathrm{C} 14.120 \pm 50 \mathrm{BP}($ Beta -311032$)$, y las correlaciones efectuadas podemos afirmar que los hallazgos corresponden al Pleistoceno final (EI 2). Desde un punto de vista biostratigráfico estos hallazgos se refieren a la biozona de Equus (Amerhippus) neogeus de Cione \& Tonni (1999, 2001). Diversas líneas de evidencias sugieren que el avance cíclico y el retroceso de los glaciares durante el Pleistoceno final producen una notable oscilación entre condiciones áridas y húmedas para la región Pampeana. Estas oscilaciones son en parte responsables de cambios en la composición de las faunas pleistocenas, expandiendo o contrayendo las zonas de distribución de los taxones y consecuentemente los límites entre la distribución de los biomas (Pascual et al., 1996). Para esta región se ha interpretado que el Lujanense comienza con condiciones interglaciales (EI 5e, 130 ka AP) en tanto que la parte final del Lujanense se desarrolló coincidentemente con el Último Máximo Glacial (18 ka AP) e incluye el último avance glacial (13 -11 ka AP), predominando en este lapso una fauna adaptada a condiciones áridas y frías (Tonni et al., 1999, 2003). Este escenario es compatible con la coexistencia de Hippidion principale e Hippidion devillei en estas latitudes.

\section{AGRADECIMIENTOS}

Queremos agradecer a Jorge Carbonari y Roberto Huarte del LATyR la realización de algunas de las dataciones radiocarbónicas. A Eduardo P. Tonni por el apoyo financiero a los trabajos de campo y de algunos de los fechados. También quere- 
mos agradecer los comentarios de Martín Ubilla que han mejorado considerablemente el manuscrito, así como el de un revisor anónimo. Este trabajo se ha realizado en el marco de los Proyectos CGL2010-19116/BOS de la DGCYT y A/023681/09 y A/030111/10 de la AECID, España, y las Subvenciones de la Universidad Nacional del Centro de la Provincia de Buenos Aires, CONICET y ANPCYT (PICT 07-01563, PICT-20110561) a JLP.

\section{Referencias}

Alberdi, M.T. (1987). La Familia Equidae, Gray 1821 (Perissodactyla, Mammalia) en el Pleistoceno de Sudamérica. IV Congreso Latinoamericano de Paleontología, Santa Cruz de la Sierra, 484-499.

Alberdi, M.T. \& Prado, J.L. (1993). Review of the genus Hippidion Owen, 1869 (Mammalia, Perissodactyla) from the Pleistocene of South America. Zoological Journal of the Linnean Society, 108: 1-22. doi:10.1111/j.1096-3642.1993.tb02559.x

Alberdi, M.T. \& Prado, J.L. (1998). Comments on: Pleistocene horses from Tarija, Bolivia, and validity of the genus Onohippidium (Mammalia: Equidae), by B. J. MacFadden. Journal of Vertebrate Paleontology, 18(3): 669-672. doi:10.1080/02724634.1998.10011094

Alberdi, M.T. \& Prado, J.L. (2004). Caballos fósiles de América del Sur. Una historia de tres millones de años. Incuapa, serie monográfica 3, 269 pp.

Alberdi M. T.; Cartelle, C. \& Prado, J.L. (2003). El registro Pleistoceno de Equus (Amerhippus) e Hippidion (Mammalia, Perissodactyla) de Brasil. Consideraciones paleoecológicas y biogeográficas. Ameghiniana, 40: 173-196.

Alberdi, M.T.; Prado, J.L. \& Favier-Dubois, C.M. (2006). Nuevo registro de Hippidion principale (Mammalia, Perissodactyla) del Pleistoceno de Mar del Sur, Argentina. Revista Española de Paleontología, 21: 105-113.

Alberdi M. T.; Zárate, M. \& Prado, J.L. (2001). Presencia de Hippidion principale en los acantilados costeros de Mar del Plata (Argentina). Revista Española de Paleontología, 16: 1-7.

Ameghino, F. (1889). Contribución al conocimiento de los mamíferos fósiles de la República Argentina. Actas Academia Nacional de Ciencias, Córdoba 6(1): 1027.

Ameghino, F. (1909). Le Diprothomo platensis: un precurseur de l'homme du Pliocene inférieur de Buenos Aires. Anales de Museo Nacional de Historia Natural de Buenos Aires, 19: 107-209.

Barnosky, A.D. \& Lindsay, E.L. (2010). Timing of Quaternary megafaunal extinction in South America in relation to human arrival and climate change. Quaternary International, 217: 10-29. doi:10.1016/j.quaint. 2009.11.017

Bidart, S. (1988). Estudio sedimentológico y mineralógico de los materiales parentales de los suelos no aluviales de la cuenca del río Sauce Grande, provincia de Buenos Aires. CONICET, unpublished Report.
Bonadonna, F.P.; Leone, G. \& Zanchetta, G. (1995). Composición isotópica de los fósiles de gasterópodos continentales de la provincia de Buenos Aires. Indicaciones paleoclimáticas. En: Evolución Biológica y Climática de la Región Pampeana durante los últimos cinco millones de años (M.T. Alberdi, G. Leone \& E.P. Tonni eds.). Monografía del Museo Nacional de Ciencias Naturales, Madrid, 12: 77-104.

Carbonari, J.E.; Huarte, R. \& Figini, A.J. (1992). Miembro guerrero, Formación Lujan (Pleistoceno, Pcia. Buenos Aires) edades 14C. Terceras Jornadas Geológicas Bonaerenses Actas 1: 245-247.

Cione, A.L. \& Tonni, E.P. (1995). Chronostratigraphy and "Land mammal-ages": the Uquian problem. Journal of Paleontology, 69: 135-159.

Cione, A.L. \& Tonni, E.P. (1999). Biostratigraphy and chronological scale of upper-most Cenozoic in the Pampean Area, Argentina. Quaternary of South America and Antarctica Peninsula, 12: 23-51.

Cione, A.L. \& Tonni, E.P. (2001). Correlation of Pliocene to Holocene southern South American and European vertebrate - bearing units. En: Neogene and Quaternary continental stratigraphy and mammal evolution (L. Rook y D. Torre eds.). Bolletino Società Paleontologica Italiana, 40(2): 167-173.

Cione, A.L. \& Tonni, E.P. (2005). Bioestratigrafía basada en mamíferos del Cenozoico superior de la provincia de Buenos Aires. $16^{\circ}$ Congreso Geológico Argentino (La Plata), RelatoRío 11: 183-200.

Cione, A.L.; Tonni, E.P.; Bargo, S.; Bond, M.; Candela, A.M.; Carlini, A.A.; Deschamps, C.M.; Dozo, M.T.; Esteban, G.; Goin, F.J.; Montalvo, C.I.; Nasif, N.; Noriega, J.I.; Ortiz Jaureguizar, E.; Pascual, R.; Prado, J.L.; Reguero, M.A.; Scillato-Yané, G.J.; Soibelzon, L.; Verzi, D.H.; Vieytes, E.C.; Vizcaino, S.F. \& Vucetich, M.G. (2007). Mamíferos continentales del Mioceno tardío a la actualidad en la Argentina: cincuenta años de estudios. Ameghiniana, $50^{\circ}$ aniversario: 257278.

Clapperton, C. (1993). Quaternary geology and geomorphology of South America. Elseiver, Amsterdam, 779 pp.

Dillon, A. \& Rabassa, J. (1985). Miembro La Chumbiada, Formación Luján (Pleistoceno, provincia de Buenos Aires): una nueva unidad estratigráfica del valle del río Salado. Actas de las I Jornadas Geológicas Bonaerenses, 1: 1-27.

Eisenmann, V.; Alberdi, M.T.; De Giuli, G. \& Staesche, U. (1988). Collected papers after the "New York International Hipparion Conference, 1981". En: Studying fossil horses, 1: Methodology (M. Woodbrune \& P. Sondaar eds.). E.J. Brill, Leiden, p. 1-72.

Fidalgo, F.; De Francesco, F. \& Colado, U. (1973). Geología superficial en las Hojas Castelli, J.M. Cobo y MonasteRío (Pcia. de Buenos Aires). $5^{\circ}$ Congreso Geológico Argentino, Actas 4: 27-39.

Fidalgo, F.; De Francesco, F. \& Pacual, R. (1975). Geología superficial de la llanura bonaerense. RelatoRío: Geología de la Provincia de Buenos Aires. Sexto Congreso Geológico Argentino, 103-138. 
Fidalgo, F.; Riggi, J.C.; Gentile, R.; Correa, H. \& Porro, N. (1991). Los "Sedimentos postpampeanos" continentales en el ámbito sur bonaerense. Revista de la Asociación Geológica Argentina, 46: 239-256.

Figini, A.; Fidalgo, F.; Huarte, R.; Carbonari, J. \& Gentile, R. (1995). Cronología radiocarbónica de los sedimentos de la Formación Luján en Arroyo Tapalqué, provincia de Buenos Aires. Actas de las Cuartas Jornadas Geológicas y Geofísicas Bonaerenses, 1:119-24.

Fucks, E.; Huarte, R.; Carbonari, J. \& Figini, A. (2007). Geocronología, paleoambientes y paleosuelos holocenos en la Región Pampeana. Revista Asociación Geológica Argentina, 62: 425-433.

Fucks, E.; Huarte, R.; Carbonari, J. \& Pisano, F. (2009). Cronología de los depósitos fluviales Pleistoceno tardío - Holoceno de la cuenca inferior y media del río Salado, provincia de Buenos Aires. IV Congreso Argentino de Cuaternario y Geomorfología; XII Congresso da Associação Brasileira de Estudos do QuaternáRío; II Reunión sobre el Cuaternario de América del Sur (La Plata) Resúmenes: 361.

Gardenal, M. (1986). Geomorfología del partido de Saliquelló, provincia de Buenos Aires. CIC Unpublished Report, 60 pp.

Gervais, P. (1855). Recherches sur les Mammifères fossils de l'Amérique méridionale. Chez P. Bertrand, Libraire-Editeur, 1-63. Paris.

Gray, J.E. (1821). On the natural arrangement of vertebrate animals. London Medical Reposit Review, 15: 296-310.

Isla, F.; Rutter, N.; Schnack, E. \& Zárate, M.A. (2000). La trasgresión Belgranense en Buenos Aires. Una revisión a cien años de su definición. Cuaternario y Ciencias Ambientales, 1: 3-14.

Lund, P.W. (1846). Meddlelse af det Udbytte de I 1844 undersögte Knoglehuler Have avgivet til hundskaben om Brasiliens Dyreverden för sidste Jordomvaeltning. Det köngelige Danske Videnskabernes Selskabs Naturvidenskabelige og Mathematiske Afhandlinger, 12: 57-94.

MacFadden, B.J. (1997). Pleistocene horses from Tarija, Bolivia, and validity of the genus Onohippidium (Mammalia, Equidae). Journal of Vertebrate Paleontology, 17: 199-218. doi:10.1080/02724634.1997.10010964

MacFadden, B.J. (1998). Preorbital Facial Fossae Onohippidium, and origin of South American Pleistocene Horses: response to Alberdi and Prado. Journal of Vertebrate Paleontology, 18(3): 673-675. doi:10.1080/02724634.1998.10011095

Moreno, F.P. (1891). Onohippidium muñizi: Breve noticia sobre los restos fósiles de un género nuevo de la familia de los Équidos conservados en el Museo de La Plata. Revista del Museo de La Plata, 2: 65-71.

Owen, R.(1848). Description of teeth and portions of jaws of two extinct anthracotherioid quadrupeds ...discovered.... in the Eoecene deposits on the NW coast of the Isle Of Wight. Quarterly Journal Geological Society, London, 4: 103-141. doi:10.1144/ GSL.JGS.1848.004.01-02.21
Owen, R. (1869). On fossil teeth of equines from Central and South America, referable to Equus conversidens, Equus tau, and Equus arcidens. Proceedings of the Royal Society of London, 17: 267-268. doi:10.1098/ rspl.1868.0042

Pascual, R.; Ortega Hinojosa, E.J.; Gondar, D. \& Tonni, E. (1965). Las edades del cenozoico mamalífero de la Argentina, con especial atención en aquellas del territoRío bonaerense. Anales y Comunicaciones de Investigación Científica, Prov. Buenos Aires 6: 165-193.

Pascual, R.; Ortega Hinojosa, E.J.; Gondar, D. \& Tonni, E.P. (1966). Las edades del Cenozoico mamalífero de la provincia de Buenos Aires. En: Paleontología Bonaerense (A.V. Borrello, ed.). Comisión de Investigaciones Científicas de la Provincia de Buenos Aires, pp. 3-12.

Pascual, R. \& Ortiz Jaureguizar, E. (1990). Evolving climates and mammal faunas in Cenozoic South American. Journal of Human Evolution, 19: 23-60. doi:10.1016/0047-2484(90)90011-Y

Pascual, R.; Ortiz Jaureguizar, E.\&y Prado, J.L. (1996). Land mammals: paradigm for Cenozoic South American geobiotic evolution. En: Contribution of Southern South America to Vertebrate Paleontology (A. Arratia, ed.). Münchner, Geowissenschaftlich Abhandlungen (A) 30: 265-319.

Pomi, L.H. (2009). Entrampamientos en la Formación Luján (Pleistoceno tardío). Análisis tafonómico de un espécimen de Hippidion Owen de la cuenca del río Salado (provincia de Buenos Aires, Argentina) Ameghiniana, 46(1): 17-25.

Pomi, L.H. \& Scanferla, C. (2008). Tafonomía sobre asociaciones de vertebrados registradas en trampas fluviales de la Región Pampeana (Buenos Aires, Argentina). Estudios Geológicos, 64: 187-196.

Prado, J.L. \& Alberdi, M.T. (1996). A cladistic analysis of the Horses of the tribe Equini. Palaeontology, 39: 663-680.

Prado, J.L. \& Alberdi, M.T. (1999). The mammalian record and climatic change over the last 13,000 years in the Pampean Region, Argentina. Quaternary International, 57/58: 165-174. doi:10.1016/S10406182(98)00057-3

Prado, J.L. \& Alberdi, M.T. (2010). Quaternary mammalian faunas of the Pampean Region. Quaternary International, 212: 176-186. doi:10.1016/j.quaint.2009.03.010

Prado, J.L.; Alberdi, M.T.; Azanza, B. \& Sánchez, B. (2001). Climate and changes in mammal diversity during the late Pleistocene-Holocene in the Pampean Region (Argentina). Acta Palaeontologica Polonica, 46(2): 261-276.

Prado, J.L.; Alberdi, M.T. \& Di Martino, V.J. (2012). Équidos y Gomphotherios del Pleistoceno tardío de las localidades de "Cascada Paleolama" y "El Conglomerado", Sudeste de la Provincia de Buenos Aires. Ameghiniana, 49(4): 623-641. doi:10.5710/ AMGH.28.4.2011.559

Prado, J.L.; Menegaz, A.; Tonni, E.P. \& Salemme, M. (1987). Los mamíferos de la fauna local de Paso Otero (Pleistoceno tardío), provincia de Buenos Aires. 
Aspectos paleoambientales y biostratigráficos. Ameghiniana, 24: 217-233.

Reig, O. 1957. Un mustélido del género Galictis del Eocuaternario de la provincia de Buenos Aires. Ameghiniana, 1: 33-47.

Scanferla, A.C.; Pomi, L.H.; Bonini, R.A.; Fucks, E. \& Molinari, A.E. (2011). A carnivore-herbivore megafauna assemblage from the Late Pleistocene of the pampas of southern South America. XVIII INQUA Congress 2011 (Berna Suiza).

Schnack, E.J. (1987). The emerged Quaternary shorelines in Argentina. Eustatic signature and correlations: Atlantic South Ocean. Resumenes Cuaternario de América del Sur, Reunión Final. Proyecto 201 (IGCPUNESCO), Ushuaia, pp. 2-6.

Schnack, E., Isla, F., De Francesco, F., y Fucks, E. (2005).ía del Cuaternario Marino Tardío en la Provincia de BuenosAires. Relatorio. XVI Congreso Geológico Argentino, La Plata, 10:-182.

Simpson, G.G. (1940). Review of the mammal-bearing tertiary of South America. Proceedings of the American Philosophical Society, 83: 649-710.

Simpson, G.G. (1971). The evolution of marsupials in South America. Annais de la Academia Brasileira de Ciências, 43: 103-118.

Toledo, M.J. (2005). Secuencias pleistocenas "lujanenses" en su sección tipo: Primeras dataciones $14 \mathrm{C}$ e implicancias estratigráficas, arqeológicas e históricas, Luján-Jáuregui, provincia de Buenos Aires. Revista de la Asociación Geológica Argentina, 60: 417-424.

Tonni, E.P. \& Cione, A.L. (1994). Los mamíferos y el clima en el Pleistoceno y Holoceno de la provincia de
Buenos Aires. Actas Jornadas Arqueológicas Interdisciplinas. Programa de Estudios Prehistóricos, Buenos Aires, 127-142.

Tonni, E.; Cione, A. \& Figini, A. (1999). Predominance of arid climates indicated by mammals in the pampas of Argentina during the Late Pleistocene and Holocene. Palaeogeography, Palaeoclimatology, Palaeoecology, 147: 257-281. doi:10.1016/S0031-0182(98)00140-0

Tonni, E.P.; Huarte, R.A.; Carbonari, J.E. \& Figini, A.J. (2003). New radiocarbon chronology for the Guerrero Member of the Luján Formation (Buenos Aires, Argentina): palaeoclimatic significance. Quaternary International, 109-110: 45-48. doi:10.1016/S10406182(02)00201-X

Zárate, M. \& Blasi, A. (1991). Late Pleistocene and Holocene loess deposits of the southereastern Buenos Aires province, Argentina. Geojournal, 24(2): 211-220. doi:10.1007/BF00186018

Zárate, M. \& Blasi, A. (1993). Late Pleistocene-Holocene Eolian deposits of the Southern Buenos Aires province, Argentina: a preliminary model. Quaternary International, 17: 15-20. doi:10.1016/10406182(93)90075-Q

Zárate, M. \& Flegenheimer, N. (1991). Geoarchaeology of the Cerro La China locality (Buenos Aires, Argentina): site 2 and site 3. Geoarchaeology, 6: 273-294. doi:10.1002/gea.3340060304

Recibido el 23 de marzo de 2012 Aceptado el 7 de septiembre de 2012 Publicado online el 15 de abril de 2013 